\title{
Adesão ao Programa de Educação Permanente para médicos de família de um Estado da Região Sudeste do Brasil
}

\author{
Adherence to the Ongoing Education Program for family doctors \\ in a southeastern Brazilian state
}

Luciana Souza d'Ávila ${ }^{1}$

Lucília Nunes de Assis ${ }^{1}$

Marilene Barros de Melo ${ }^{1}$

Luiz Carlos Brant ${ }^{2}$
${ }^{1}$ Superintendência de Pesquisa (SUPE), Escola de Saúde Pública de Minas Gerais. Av. Augusto de Lima 2061/210, Barro Preto. 30.190-002 Belo Horizonte MG Brasil.

lucianasd14m@yahoo.com.br ${ }^{2}$ Universidade Federal de Minas Gerais.
Abstract Ongoing Health Education is a strategy for transformation of health practices, though the adherence of professionals is one of the challenges facing its implementation. Thus, the objective of this study was to investigate the factors associated with adherence of family doctors to the Ongoing Education Program in a southeastern Brazilian state from the perception of supervisors. It is a cross-sectional and quantitative study with the use of online questionnaires. Data were analyzed using the chi-square test with ongoing correction to determine the association between structure, topics, activities and difficulties of the supervisors working in Ongoing Health Education, difficulties of the physicians in Primary Health Care (PHC) and poor and good adherence to the program. Excellent medical participation was statistically related to the adequacy of physical space ( $p$ $=0.001)$, a multidisciplinary approach $(p=0.035)$ and epidemiological aspects $(p=0.043)$. Low adherence was associated with the inadequacy of the physical structure, difficulty understanding the methodology, less time in a supervisory position, multiple workdays, among others. A good adherence to Ongoing Health Education is a possibility for collective reconstruction of the everyday work of physicians in Primary Health Care.

Key words Ongoing education, Primary Health Care, Medical education
Resumo A Educação Permanente em Saúde é uma estratégia de transformação das práticas em saúde, sendo a adesão do profissional um dos desafios para sua efetivação. Nessa perspectiva, o objetivo deste trabalho foi investigar, a partir da percepção dos supervisores, os fatores relacionados à adesão dos médicos às atividades do Programa de Educação Permanente (PEP) para médicos de família de um Estado da região Sudeste. Tratase de um estudo transversal, quantitativo, com utilização de questionários on-line. Os dados foram analisados por meio do teste qui-quadrado com correção de continuidade para se verificar a associação entre a estrutura, tópicos, atividades do PEP, dificuldades dos supervisores no trabalho no PEP e dos médicos na APS e a baixa e boa adesão ao Programa. A ótima participação dos médicos se relacionou à adequação do espaço físico $(p=0,001)$ e à abordagem multidisciplinar ( $p$ $=0,035)$ e dos aspectos epidemiológicos $(p=0,043)$. Já a baixa adesão se associou à inadequação da estrutura física, à dificuldade de compreensão da metodologia, ao menor tempo de atuação do supervisor, às múltiplas jornadas de trabalho dos médicos, entre outras. A boa adesão ao PEP é uma possibilidade de reconstrução coletiva da realidade laboral cotidiana e da prática médica na Atenção Primária em Saúde.

Palavras-chave Educação permanente, Atenção Primária à Saúde, Educação médica 


\section{Introdução}

O Programa de Saúde da Família (PSF) foi criado em 1994 como eixo reorientador do modelo de atenção no âmbito do Sistema Único de Saúde (SUS), sendo que, em 1997, foi redefinido como Estratégia de Saúde da Família (ESF). A operacionalização dessa estratégia se dá a partir do trabalho de equipes multiprofissionais, que são constituídas, no mínimo, por um auxiliar de enfermagem, seis agentes comunitários de saúde (ACS), um enfermeiro e um médico generalista. Esses profissionais são responsáveis por grupos populacionais de aproximadamente mil famílias, ou quatro mil habitantes, em territórios delimitados $^{1}$

Diante do desafio imposto pelas diretrizes da ESF, pelos saberes que mediam a relação equipe/ comunidade e entre os integrantes da equipe, e pela necessidade de adequação às especificidades de cada território, são necessários mudanças no modelo de formação ${ }^{2}$, bem como, investimentos nos processos permanentes de formação e qualificação profissionais. Nesse contexto, a Portaria GM/MS no 1.996, de 20 de agosto de 2007, implementa a Política Nacional de Educação Permanente em Saúde ${ }^{3}$. Alguns movimentos procuram consolidar essa política, entre eles, o Programa de Educação Permanente (PEP) para médicos de Saúde da Família de um Estado da região sudeste.

O PEP, lançado em 2004, é financiado integralmente com recursos do tesouro estadual e está presente em todas as 13 regiões ampliadas de saúde do Estado investigado, tendo como parceiras 17 instituições ( 16 universidades e a prefeitura da capital do Estado). A opção desse programa em contemplar, inicialmente, somente os médicos da Saúde da Família se relaciona à baixa adesão desses profissionais à ESF, com curta permanência nas equipes; à frágil formação do médico no campo da Atenção Primária, assim como à pequena participação desses trabalhadores em qualificações pertinentes a esse nível de atenção ${ }^{4}$.

De acordo com Silvério4, o objetivo da implementação do PEP foi favorecer o exercício da prática médica de maneira a reconstruí-la, permanentemente, de acordo com as necessidades em saúde, visando o aprimoramento do cuidado. Entre as suas metas, encontram-se a qualificação da prática; o fortalecimento da autoestima; a minimização do isolamento; o estabelecimento de protocolos; a ampliação da efetividade e resolutividade da APS e a redução dos diagnósticos e prescrições incorretos e da rotatividade dos médicos na ESF$F^{4}$.
Em relação à sua metodologia, o PEP tem como núcleo a consulta médica centrada no paciente e é organizado a partir dos Grupos de Aperfeiçoamento Profissional (GAP), compostos por 8 a 12 médicos que integram a ESF de uma mesma região de saúde. As atividades desses grupos são desenvolvidas em encontros mensais ou quinzenais, com a orientação de um professor da Universidade parceira, ou de um médico de família pertencente à rede de um dos municípios da região. Esse professor/médico de família é chamado de supervisor e é responsável por direcionar e acompanhar as discussões dos GAP, de acordo com a metodologia preconizada pelo programa, sem aulas expositivas ${ }^{4}$.

Quanto à infraestrutura do programa, recomenda-se que seja disponibilizado nos municípios um espaço educativo constituído por salas de educação permanente, com capacidade e qualidade adequadas. Ainda, o PEP é operacionalizado por meio de quatro estratégias educacionais, sendo que para cada, devem ser dedicadas quatro horas mensais no horário de trabalho, organizadas de acordo com as necessidades de cada grupo ${ }^{4}$.

Essas estratégias educacionais englobam: a) Plano de Desenvolvimento Profissional (PDP) ferramenta para promover a autoaprendizagem e a identificação das fragilidades profissionais individuais, sobre as quais são traçadas estratégias; b) Módulos de Capacitação - reuniões em torno de um eixo temático constituído a partir do estabelecimento de prioridades, identificadas pelos gestores e médicos do GAP; c) Treinamento de Habilidades Clínicas - acompanhamento e avaliação de um preceptor/ facilitador quanto ao atendimento clínico; e, d) Ciclo de Aperfeiçoamento de Prática Profissional (CAPP) - pilar da ação educacional, constituindo um espaço permanente de discussão e de proposição crítica da prática, por meio da análise das consultas, dos eventos, dos prontuários, dos mecanismos de auditoria clínica e operacional, da discussão de casos clínicos e de protocolos externos e internos ${ }^{4,5}$.

O referencial teórico do Programa, por sua vez, refere-se à Educação Permanente em Saúde (EPS), que pode ser compreendida como uma estratégia de desenvolvimento de ações educativas que tem seu significado traduzido no processo de trabalho. De acordo com esse referencial, o cotidiano laboral se configura como fonte de conhecimento, propiciando a reflexão e a problematização da realidade em saúde, a partir da "experienciação", de maneira a compreendê-la e, se necessário, propor ações para transformá-la. 
Assim, a construção da ação educativa deve estar pautada nas necessidades de saúde e especificidades locais, além de ser articulada ao processo de atenção, à formação/qualificação, à gestão e ao controle social ${ }^{6-10}$.

A importância da Educação Permanente na Atenção Primária em Saúde está associada à busca pela garantia da universalidade e integralidade do SUS, uma vez que, além de compreender um território adscrito a partir do enfoque familiar e comunitário, a APS é considerada espaço de construção coletiva, onde os diversos sujeitos estão envolvidos nos cuidados em saúde ${ }^{8,11,12}$.

Diante da complexidade da ação que o PEP se propõe, fez-se necessário analisar a sua implantação. O objetivo deste trabalho é identificar, a partir da percepção dos supervisores, os fatores relacionados à adesão dos médicos às atividades do Programa de Educação Permanente para médicos de família de 11 regiões ampliadas de saúde em um Estado da Região Sudeste.

\section{Metodologia}

\section{Obtenção das informações}

Trata-se de um estudo transversal, de natureza quali-quantitativa, realizada entre abril de 2011 e janeiro de 2013, que teve como objetivo analisar o processo de implementação do PEP. Este artigo tem como foco os fatores relacionados à adesão (participação, assiduidade, frequência e envolvimento) dos médicos nas atividades do programa, segundo a percepção dos supervisores dos GAP.

As informações foram obtidas a partir da aplicação de um questionário on-line ao universo de supervisores que iniciaram sua atuação no programa até agosto de $2011(\mathrm{~N}=215)$. Foi realizado um piloto com três representantes desses atores, visando à compreensão e a pertinência do conteúdo do questionário, assim como da operacionalização e utilização da ferramenta web (LimeSurvey ${ }^{\circledR}$ ) utilizada para envio do instrumento.

Paralelamente, os endereços eletrônicos e/ou números de telefone dos supervisores foram coletados por meio do contato com os coordenadores da prefeitura e das Universidades parceiras. O instrumento de coleta foi hospedado na página da Escola de Saúde Pública do Estado e o link codificado, de acesso individual, foi enviado ao endereço eletrônico dos participantes $(\mathrm{N}=$ 215). A utilização do questionário visou à construção do perfil dos supervisores, obtenção de informações quanto às ações e atividades do programa e sua concepção sobre a adesão dos médicos e a prática na APS.

Após o encaminhamento dos questionários, foram enviadas três mensagens lembrando a importância da resposta, em intervalos de 10 a 15 dias, além de novo envio em caso de solicitação individual pelo supervisor. O prazo de resposta foi de dois meses, sendo ampliado por mais dois, devido a problemas operacionais com o servidor de rede da instituição executora da pesquisa.

O questionário respondido não apresentou qualquer tipo de identificação e continha duas formas de consentimento ou recusa de participação na pesquisa. A primeira forma consistiu em um recurso automático do programa web, em que, no corpo do e-mail enviado, foram disponibilizados dois links distintos, sendo o primeiro de aceite de participação e, o segundo, de recusa. Ao clicar no link de aceite, o supervisor tinha acesso ao questionário, cuja primeira pergunta foi denominada de "Termo de Consentimento Livre e Esclarecido" (segunda forma de consentimento ou recusa), contendo explicações gerais sobre o estudo, assim como sobre a garantia de anonimato das respostas. Essa questão englobou duas opções “sim” ou "não”, sendo que ao marcar a resposta "não", o supervisor foi excluído do estudo.

A pesquisa foi submetida e aprovada pelo Comitê de Ética do Centro de Pesquisa René Rachou.

\section{Análise das informações}

Para análise das informações foi utilizado o desenho de estudo do tipo caso-controle, realizando-se, primeiramente, uma análise descritiva das informações referentes ao perfil dos supervisores. A seguir, fez-se o cruzamento entre as variáveis relacionadas à participação, frequência, assiduidade e envolvimento dos médicos nas atividades do programa (variáveis resposta) e as variáveis relativas às ações do programa e à prática dos médicos na APS (variáveis explicativas).

As variáveis explicativas foram categorizadas em: a) estrutura do Programa; b) critérios considerados na organização e desenvolvimento dos GAP; c) tópicos abordados e atividades desenvolvidas no PEP; d) atuação dos supervisores e dificuldades no cotidiano laboral; e) percepção dos supervisores das dificuldades dos médicos na APS. As variáveis resposta foram classificadas em: a) boa adesão ao PEP e b) baixa adesão (Quadro 1).

Para verificar a associação entre as variáveis resposta e explicativas, utilizou-se o teste qui- 
Quadro 1. Variáveis explicativas e variáveis resposta utilizadas na análise das informações, Programa de Educação Permanente para Médicos de Família, Sudeste, Brasil, 2011-2012.

\begin{tabular}{|c|c|c|}
\hline & & Variáveis \\
\hline \multirow{5}{*}{ 空 } & $\begin{array}{l}\text { A) Estrutura do } \\
\text { Programa }\end{array}$ & $\begin{array}{l}\text { a) Adequação do espaço físico às demandas dos GAP: ÓTIMA } \\
\text { b) Adequação do espaço físico às demandas dos GAP: BOA } \\
\text { c) Adequação do espaço físico às demandas dos GAP: REULAR } \\
\text { d) Adequação do espaço físico às demandas dos GAP: RUIM }\end{array}$ \\
\hline & $\begin{array}{l}\text { B) Critérios considerados } \\
\text { na organização e } \\
\text { desenvolvimento dos } \\
\text { GAP }\end{array}$ & $\begin{array}{l}\text { a) Conteúdos pré-programados } \\
\text { b) Demandas individuais dos médicos } \\
\text { c) Demandas discutidas e consensualizadas pelo grupo } \\
\text { d) Diagnóstico da situação de saúde da população } \\
\text { e) Prioridades dos gestores municipais de saúde }\end{array}$ \\
\hline & $\begin{array}{l}\text { C) Tópicos abordados e } \\
\text { atividades desenvolvidas } \\
\text { no PEP }\end{array}$ & $\begin{array}{l}\text { a) Abordagem clínica dos problemas de saúde prevalentes na APS } \\
\text { b) Aspectos epidemiológicos dos problemas de saúde prevalentes na APS } \\
\text { c) Abordagem multidisciplinar dos problemas de saúde prevalentes na APS } \\
\text { d) Organização das ações e serviços da Unidade de Saúde } \\
\text { e) Referência e contrarreferência } \\
\text { f) Habilidade em comunicação e educação em saúde - relação médico- } \\
\text { usuário } \\
\text { g) Habilidade em comunicação e educação em saúde - abordagem coletiva } \\
\text { h) Aprimoramento da consulta médica e dos métodos clínicos centrados } \\
\text { no paciente } \\
\text { i) Discussão de casos clínicos } \\
\text { j) Discussão de eventos críticos }\end{array}$ \\
\hline & $\begin{array}{l}\text { D) Atuação dos } \\
\text { supervisores e suas } \\
\text { dificuldades no } \\
\text { cotidiano de trabalho no } \\
\text { PEP }\end{array}$ & $\begin{array}{l}\text { a) Tempo de atuação no PEP } \\
\text { b) Dificuldade de acesso a material de referencial teórico } \\
\text { c) Dificuldade com espaço físico utilizado } \\
\text { d) Dificuldade de disponibilidade de materiais e insumos } \\
\text { e) Dificuldade de disponibilidade e qualidade de equipamentos e } \\
\text { instrumentos } \\
\text { f) Dificuldade de articulação e cooperação por parte da instituição de } \\
\text { ensino } \\
\text { g) Dificuldade de articulação e cooperação por parte da gestão regional } \\
\text { h) Dificuldade de articulação e cooperação por parte da gestão municipal } \\
\text { i) Dificuldade de compreensão e adesão dos médicos à metodologia } \\
\text { utilizada no PEP } \\
\text { j) Dificuldade com a metodologia utilizada pelo PEP }\end{array}$ \\
\hline & $\begin{array}{c}\text { E) Percepção dos } \\
\text { supervisores quanto às } \\
\text { dificuldades dos médicos } \\
\text { na APS }\end{array}$ & $\begin{array}{l}\text { a) Múltiplas jornadas de trabalho } \\
\text { b) Remuneração insatisfatória } \\
\text { c) Manejo clínico } \\
\text { d) Encaminhamento aos serviços de referência } \\
\text { e) Organização das ações e serviços da Unidade de Saúde } \\
\text { f) Trabalho em equipe } \\
\text { g) Espaço físico inadequado } \\
\text { h) Materiais e insumos insuficientes } \\
\text { i) Equipamentos e instrumentos insuficientes e/ou inadequados }\end{array}$ \\
\hline \multirow{2}{*}{ 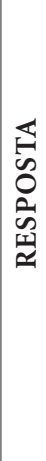 } & A) Boa adesão ao PEP & $\begin{array}{l}\text { a) Participação dos médicos nas atividades do PEP: BOA } \\
\text { b) Participação dos médicos nas atividades do PEP: ÓTIMA } \\
\text { c) Assiduidade dos médicos nas atividades do PEP: BOA } \\
\text { d) Assiduidade dos médicos nas atividades do PEP: ÓTIMA }\end{array}$ \\
\hline & B) Baixa adesão ao PEP & $\begin{array}{l}\text { a) Participação dos médicos nas atividades do PEP: REGULAR } \\
\text { b) Participação dos médicos nas atividades do PEP: RUIM } \\
\text { c) Assiduidade dos médicos nas atividades do PEP: REGULAR } \\
\text { d) Assiduidade dos médicos nas atividades do PEP: RUIM } \\
\text { e) FREQUÊNCIA dos médicos é dificuldade do cotidiano de trabalho no } \\
\text { PEP } \\
\text { f) ENVOLVIMENTO dos médicos é dificuldade do cotidiano de trabalho } \\
\text { no PEP }\end{array}$ \\
\hline
\end{tabular}


quadrado com correção de continuidade, a uma significância $\mathrm{p} \leq 0,05$ e o cálculo da razão de chances (RC) e de seu intervalo de confiança (IC) a 95\%. Para a realização dos testes descritos foi utilizado o Pacote Estatístico SPSS 19,0.

\section{Resultados}

\section{Perfil dos supervisores}

Foi obtida uma taxa de resposta de 71,6\% (n $=154$ ), considerada boa em relação às esperadas relatadas na literatura ${ }^{13,14}$ para estudos de avaliação de necessidades e de impacto e com populações homogêneas em determinadas características, no caso, ser supervisor do PEP.

O perfil dos supervisores é equitativo quanto ao gênero, sendo $49,4 \%(n=76)$ dos participantes do sexo feminino e $50,6 \%(n=78)$, do masculino. Aproximadamente, $47 \%(\mathrm{n}=72)$ dos supervisores estão na faixa etária entre 31 a 40 anos e 45,5\% ( $n=70)$ são especialistas na área de Atenção Primária, Saúde da Família, Medicina de Família ou Saúde Pública. A maioria dos supervisores tem como instituição de origem a Universidade $(38,3 \%$; 59$)$ ou Serviços de Saúde (37,7\%; $58)$ e $13,6 \%(n=21)$ são originados de ambas as instituições. A experiência na assistência à saúde pelos médicos supervisores é predominante na APS, referenciada por $86,4 \%$ (Tabela 1 ).

\section{Fatores relacionados à boa adesão às atividades do PEP}

A boa adesão dos médicos de família foi verificada pela análise dos fatores associados à $\mathrm{BOA}$ ou ÓTIMA participação e à BOA ou ÓTIMA assiduidade nas atividades do programa. Em relação à participação dos médicos, a boa participação se mostrou inversamente associada ( $\mathrm{p}=$ $0,050)$ a uma adequação ruim do espaço físico às demandas dos GAP $\left(\mathrm{RC}=0,46 ; \mathrm{IC}_{95 \%}=0,22\right.$ $0,94)$ ou seja, o fato de se ter uma adequação ruim do espaço físico, reduz em 2,17 vezes a chance de se ter uma boa participação dos médicos no PEP (Tabela 2).

De forma contrária, verificou-se que a boa adequação do espaço físico às demandas dos GAP $\left(\mathrm{p}=0,001 ; \mathrm{RC}=5,56 ; \mathrm{IC}_{95 \%}=1,94-15,90\right)$, a abordagem multidisciplinar $(\mathrm{p}=0,035 ; \mathrm{RC}=$ 3,$\left.50 ; \mathrm{IC}_{95 \%}=1,18-10,36\right)$ e dos aspectos epidemiológicos $\left(\mathrm{p}=0,043 ; \mathrm{RC}=3,60 ; \mathrm{IC}_{95 \%}=1,13-11,51\right)$ dos problemas de saúde prevalentes na APS se associaram positivamente à ótima participação nas atividades do programa. Em outras palavras, o cruzamento com a boa adequação do espaço físico apontou uma chance de 5,5 vezes de

Tabela 1. Perfil dos supervisores $(n=154)$ quanto às características demográficas, à formação e à atuação profissional, Sudeste, Brasil, 2011-2012.

\begin{tabular}{|c|c|c|}
\hline Perfil dos supervisores & $\mathbf{n}$ & $\%$ \\
\hline \multicolumn{3}{|l|}{ Características demográficas } \\
\hline \multicolumn{3}{|l|}{ Sexo } \\
\hline Feminino & 76 & 49,4 \\
\hline Masculino & 78 & 50,6 \\
\hline Total & 154 & 100,0 \\
\hline \multicolumn{3}{|l|}{ Faixa etária } \\
\hline De 21 a 30 anos & 11 & 7,1 \\
\hline De 31 a 40 anos & 72 & 46,8 \\
\hline De 41 a 50 anos & 30 & 19,5 \\
\hline 50 anos ou mais & 41 & 26,6 \\
\hline Total & 154 & 100,0 \\
\hline \multicolumn{3}{|l|}{ Formação } \\
\hline \multicolumn{3}{|l|}{ Especialização* } \\
\hline Não & 49 & 31,8 \\
\hline $\begin{array}{l}\text { APS, Saúde da Família, Medicina } \\
\text { de Família ou Saúde Pública }\end{array}$ & 70 & 45,5 \\
\hline Outras especialidades & 58 & 37,7 \\
\hline \multicolumn{3}{|l|}{ Residência $^{*}$} \\
\hline Não & 83 & 53,9 \\
\hline $\begin{array}{l}\text { APS, Saúde da Família, Medicina } \\
\text { de Família ou Saúde Pública }\end{array}$ & 23 & 14,9 \\
\hline Outras áreas & 54 & 35,1 \\
\hline \multicolumn{3}{|l|}{ Mestrado } \\
\hline Não & 115 & 74,7 \\
\hline $\begin{array}{l}\text { APS, Saúde da Família, Medicina } \\
\text { de Família ou Saúde Pública }\end{array}$ & 11 & 7,1 \\
\hline Outras áreas & 28 & 18,2 \\
\hline Total & 154 & 100,0 \\
\hline \multicolumn{3}{|l|}{ Doutorado } \\
\hline Não & 132 & 85,7 \\
\hline $\begin{array}{l}\text { APS, Saúde da Família, Medicina } \\
\text { de Família ou Saúde Pública }\end{array}$ & 03 & 1,9 \\
\hline Outras áreas & 19 & 12,3 \\
\hline Total & 154 & 100,0 \\
\hline \multicolumn{3}{|l|}{ Atuação profissional } \\
\hline \multicolumn{3}{|l|}{ Instituição de origem } \\
\hline Universidade & 59 & 38,3 \\
\hline Serviço & 58 & 37,7 \\
\hline Universidade e serviço & 21 & 13,6 \\
\hline Não especificado & 16 & 10,4 \\
\hline Total & 154 & 100,0 \\
\hline \multicolumn{3}{|l|}{ Área de experiência assistencial } \\
\hline Atenção Primária à Saúde & 133 & 86,4 \\
\hline Pronto Atendimento & 61 & 39,6 \\
\hline Urgência/Emergência & 47 & 30,5 \\
\hline Atenção especializada & 84 & 54,5 \\
\hline
\end{tabular}

Total maior que 154, pois alguns supervisores deram mais de uma resposta para essa questão. 
Tabela 2. Fatores relacionados à BOA ou ÓTIMA participação e à ÓTIMA assiduidade dos médicos nas atividades do PEP, Sudeste, Brasil, 2011-2012.

BOA participação dos médicos nas atividades do PEP

\begin{tabular}{lccccc}
\hline & Sim & Não & & \\
& $\mathbf{n}(\%)$ & $\mathbf{n}(\%)$ & $\mathbf{p}$ & $\mathbf{R C}$ & IC 95\% \\
\hline Estrutura do Programa & & & & & \\
$\quad$ Adequação do espaço físico às demandas dos GAP: & & & & & \\
RUIM & $16(36,4)$ & $28(63,6)$ & 0,050 & 0,46 & $0,22-0,94$ \\
$\quad$ Sim & $61(55,5)$ & $49(44,5)$ & & & \\
$\quad$ Não &
\end{tabular}

ÓTIMA participação dos médicos nas atividades do PEP

\begin{tabular}{|c|c|c|c|c|c|}
\hline & $\begin{array}{l}\operatorname{Sim} \\
\mathbf{n}(\%)\end{array}$ & $\begin{array}{l}\text { Não } \\
\text { n }(\%)\end{array}$ & $\mathbf{p}$ & $\mathrm{RC}$ & IC 95\% \\
\hline \multicolumn{6}{|c|}{ Estrutura do Programa } \\
\hline \multicolumn{6}{|c|}{$\begin{array}{l}\text { Adequação do espaço físico às demandas dos GAP: } \\
\text { BOA }\end{array}$} \\
\hline Sim & $12(25,0)$ & $36(75,0)$ & 0,001 & 0,46 & $1,94-15,90$ \\
\hline Não & $6(5,7)$ & $100(94,3)$ & & & \\
\hline \multicolumn{6}{|c|}{ Tópicos abordados e atividades desenvolvidas no } \\
\hline \multicolumn{6}{|c|}{$\begin{array}{l}\text { Aspectos epidemiológicos dos problemas de saúde } \\
\text { prevalentes na APS }\end{array}$} \\
\hline Sim & $14(17,3)$ & $67(82,7)$ & 0,043 & 0,46 & $1,13-11,51$ \\
\hline Não & $4(5,5)$ & $69(94,5)$ & & & \\
\hline \multicolumn{6}{|c|}{$\begin{array}{l}\text { Abordagem multidisciplinar dos problemas de } \\
\text { saúde prevalentes na APS }\end{array}$} \\
\hline Sim & $13(18,3)$ & $58(81,7)$ & 0,035 & 0,46 & $1,18-10,36$ \\
\hline Não & $5(6,0)$ & $78(94,0)$ & & & \\
\hline
\end{tabular}

ÓTIMA assiduidade dos médicos nas atividades do PEP

\begin{tabular}{cccccc}
\hline Sim & Não \\
$\mathbf{n}(\%)$ & $\mathbf{n}(\%)$ & $\mathbf{p}$ & RC & IC 95\% \\
\hline
\end{tabular}

Estrutura do Programa

Adequação do espaço físico às demandas dos GAP: BOA

Sim

Não

Tópicos abordados e atividades desenvolvidas no PEP Aspectos epidemiológicos dos problemas de saúde prevalentes na APS

Sim

Não

Atuação dos supervisores e suas dificuldades no cotidiano de trabalho no PEP

Dificuldade de compreensão e adesão dos médicos

participantes à metodologia utilizada no PEP

Sim

Não

Percepção dos supervisores quanto às dificuldades dos médicos na APS

Remuneração insatisfatória

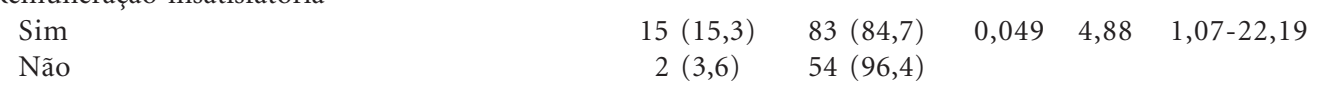

$\begin{array}{ccccc}11(22,9) & 37(77,1) & 0,004 & 4,95 & 1,71-14,36 \\ 6(5,7) & 100(94,3) & & & \\ & & & \\ & & & & \\ 15(18,5) & 66(81,5) & 0,004 & 8,07 & 1,78-36,63 \\ 2(2,7) & 71(97,3)\end{array}$

$2(3,3) \quad 59(96,7) \quad 0,026 \quad 0,18 \quad 0,04-0,80$

$15(16,1) \quad 78(83,9)$

$2(3,6) \quad 54(96,4)$ 
se ter uma ÓTIMA participação, enquanto a abordagem multidisciplinar e dos aspectos epidemiológicos dos problemas de saúde prevalentes na APS apresentaram uma chance de 3,5 e 3,6 vezes, respectivamente (Tabela 2). As outras variáveis explicativas não apresentaram uma associação estatisticamente significante com a boa ou ótima participação.

Quanto à assiduidade nas atividades do PEP, a boa assiduidade não se mostrou relacionada com nenhuma variável. Por outro lado, foi encontrada uma associação positiva da ótima assiduidade com a boa adequação do espaço físico às demandas dos GAP $(\mathrm{p}=0,004 ; \mathrm{RC}=4,95$; $\left.\mathrm{IC}_{95 \%}=1,71-14,36\right)$; com a abordagem dos aspectos epidemiológicos dos problemas de saúde prevalentes na APS ( $\mathrm{p}=0,004 ; \mathrm{RC}=8,07 ; \mathrm{IC}_{95 \%}=$ 1,78-36,63) e com a remuneração insatisfatória dos médicos na APS ( $\mathrm{p}=0,049 ; \mathrm{RC}=4,88 ; \mathrm{IC}_{95 \%}$ $=1,07-22,19)$. Uma associação inversa foi observada entre a ótima assiduidade e a dificuldade, vivenciada pelo supervisor, de compreensão e adesão dos médicos participantes à metodologia utilizada no PEP ( $\mathrm{p}=0,026 ; \mathrm{RC}=0,18 ; \mathrm{IC}_{95 \%}=$ 0,04-0,80).

Observa-se que, novamente, a adequação do espaço físico às demandas dos GAP se mostrou relacionada com a adesão às atividades do PEP, uma vez que uma boa adequação do espaço apresenta, em média, 4,95 vezes mais chance de se ter uma ótima assiduidade no programa. A abordagem dos aspectos epidemiológicos também pode ser considerada relevante, já que indica uma chance aumentada em 8,07 vezes de se ter uma ótima assiduidade no PEP. Vale ressaltar que o amplo intervalo de confiança encontrado nesse caso pode ser explicado pela presença de um valor menor que cinco na contagem dessa variável. Além disso, a dificuldade percebida pelos supervisores em seu cotidiano de trabalho no PEP de compreensão e adesão dos médicos à metodologia utilizada no programa pode reduzir em 5,5 vezes a chance de se ter uma ótima assiduidade. De forma inversa, a remuneração insatisfatória na APS, segundo a percepção dos supervisores, contribui para ótima assiduidade no programa (Tabela 2).

\section{Fatores relacionados à baixa adesão às atividades do PEP}

A baixa adesão dos médicos de família ao programa foi analisada por meio dos fatores associados à participação e assiduidade REGULAR ou RUIM dos profissionais e por meio da dificuldade, percebida pelos supervisores, de FRE-
QUÊNCIA e ENVOLVIMENTO dos médicos nas atividades do PEP.

Quanto à participação dos médicos, a adequação ruim do espaço físico às demandas do $\operatorname{GAP}\left(\mathrm{p}=0,012 ; \mathrm{RC}=2,67 ; \mathrm{IC}_{95 \%}=1,29-5,50\right)$; a abordagem da habilidade em comunicação e educação em saúde/relação médico-usuário ( $\mathrm{p}$ = 0,$\left.025 ; \mathrm{RC}=2,72 ; \mathrm{IC}_{95 \%}=1,19-6,20\right)$ e a dificuldade, vivenciada pelo supervisor, de compreensão e adesão dos médicos à metodologia utilizada ( $\mathrm{p}$ $=0,040 ; \mathrm{RC}=2,16 ; \mathrm{IC}_{95 \%}=1,09-4,27$ ), apresentaram uma relação positiva com a participação regular no programa. A não abordagem dos aspectos epidemiológicos dos problemas de saúde prevalentes na APS ( $\mathrm{p}=0,046 ; \mathrm{RC}=0,47 ; \mathrm{IC}_{95 \%}=$ $0,24-0,94)$ e a não discussão de eventos críticos $\left(\mathrm{p}=0,023 ; \mathrm{RC}=0,41 ; \mathrm{IC}_{95 \%}=0,20-0,84\right)$, também se mostraram relacionadas à participação considerada regular (Tabela 3). No caso da participação ruim, observou-se uma relação estatisticamente positiva com a dificuldade de articulação e cooperação por parte da gestão municipal ( $\left.\mathrm{p}=0,010 ; \mathrm{RC}=13,97 ; \mathrm{IC}_{95 \%}=1,58-123,37\right)$.

A abordagem multidisciplinar dos problemas de saúde prevalentes na APS mais uma vez se relacionou à adesão, já que o fato de não abordar esse tópico no programa, está associada a uma assiduidade considerada regular 2,32 vezes maior $\left(\mathrm{p}=0,020 ; \mathrm{RC}=0,43 ; \mathrm{IC}_{95 \%}=0,22-0,84\right)$. Paralelamente, um tempo de atuação do supervisor no PEP menor ou igual a um ano determinou a chance de se ter uma assiduidade regular de 2,11 vezes maior ( $\mathrm{p}=0,037$; $\left.\mathrm{RC}=2,11 ; \mathrm{IC}_{95 \%}=1,10-4,06\right)$, ou seja, um tempo de atuação do supervisor maior que um ano está associado a uma melhor assiduidade dos médicos (Tabela 3).

A assiduidade ruim dos médicos, por sua vez, apresentou uma relação inversa com a discussão de casos clínicos no PEP ( $p=0,041 ; \mathrm{RC}=0,14$; $\left.\mathrm{IC}_{95 \%}=0,03-0,74\right)$ e uma relação positiva com as múltiplas jornadas de trabalho na APS ( $\mathrm{p}=0,018$; $\left.\mathrm{RC}=4,78 ; \mathrm{IC}_{95 \%}=1,34-17,02\right)$, percebidas como dificuldades pelos supervisores (Tabela 3 ).

No caso da frequência dos médicos percebida como uma dificuldade pelos supervisores em seu cotidiano no PEP, observou-se uma relação inversa com a discussão de tópicos que envolvem a habilidade em comunicação e educação em saúde focada na abordagem coletiva $(\mathrm{p}=$ 0,$\left.050 ; \mathrm{RC}=0,44 ; \mathrm{IC}_{95 \%}=0,21-0,94\right)$. Ao se realizar o cruzamento da "frequência dos médicos" com outras variáveis da categoria de dificuldades percebidas pelos supervisores, encontrou-se uma associação positiva com a dificuldade de compreensão e adesão dos médicos à metodolo- 
Tabela 3. Fatores relacionados à participação REGULAR ou RUIM e à assiduidade REGULAR ou RUIM dos médicos nas atividades do PEP, Sudeste, Brasil, 2011-2012.

\begin{tabular}{|c|c|c|c|c|c|}
\hline \multicolumn{6}{|c|}{ Participação REGULAR dos médicos nas atividades do PEP } \\
\hline & $\begin{array}{l}\text { Sim } \\
\mathbf{n}(\%)\end{array}$ & $\begin{array}{l}\text { Não } \\
\text { n }(\%)\end{array}$ & $\mathbf{p}$ & RC & IC $(95 \%)$ \\
\hline \multicolumn{6}{|l|}{ Estrutura do Programa } \\
\hline \multicolumn{6}{|l|}{$\begin{array}{l}\text { Adequação do espaço físico às demandas dos GAP: } \\
\text { RUIM }\end{array}$} \\
\hline $\operatorname{Sim}$ & $22(50,0)$ & $22(50,0)$ & 0,012 & 2,67 & $1,29-5,50$ \\
\hline Não & $30(27,3)$ & $80(72,7)$ & & & \\
\hline \multirow{2}{*}{\multicolumn{6}{|c|}{$\begin{array}{l}\text { Tópicos abordados e atividades desenvolvidas no PEP } \\
\text { Aspectos epidemiológicos dos problemas de saúde } \\
\text { prevalentes na APS }\end{array}$}} \\
\hline & & & & & \\
\hline $\operatorname{Sim}$ & $21(25,9)$ & $60(74,1)$ & 0,046 & 0,47 & $0,24-0,94$ \\
\hline Não & $31(42,5)$ & $42(57,5)$ & & & \\
\hline \multirow{2}{*}{\multicolumn{6}{|c|}{$\begin{array}{l}\text { Habilidade em comunicação e educação em saúde } \\
\text { - relação médico-usuário }\end{array}$}} \\
\hline & & & & & \\
\hline Sim & $43(39,8)$ & $65(60,2)$ & 0,025 & 2,72 & $1,19-6,20$ \\
\hline Não & $9(19,6)$ & $37(80,4)$ & & & \\
\hline \multicolumn{6}{|l|}{ Discussão de eventos críticos } \\
\hline $\operatorname{Sim}$ & $31(27,9)$ & $80(72,1)$ & 0,023 & 0,41 & $0,20-0,84$ \\
\hline Não & $21(48,8)$ & $22(51,2)$ & & & \\
\hline \multicolumn{6}{|l|}{$\begin{array}{l}\text { Atuação dos supervisores e suas dificuldades no } \\
\text { cotidiano de trabalho no PEP }\end{array}$} \\
\hline $\begin{array}{l}\text { Dificuldade de compreensão e adesão dos méc } \\
\text { participantes à metodologia utilizada no PEP }\end{array}$ & & $34(55,7)$ & 0,040 & 2,16 & $1,09-4,27$ \\
\hline Sim & $27(44,3)$ & $68(73,1)$ & & & \\
\hline Não & $25(26,9)$ & & & & \\
\hline
\end{tabular}

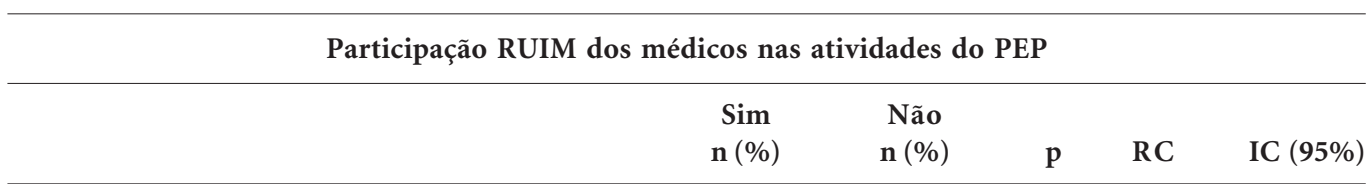

Atuação dos supervisores e suas dificuldades no cotidiano de trabalho no PEP

Dificuldade de articulação e cooperação por parte

da gestão municipal

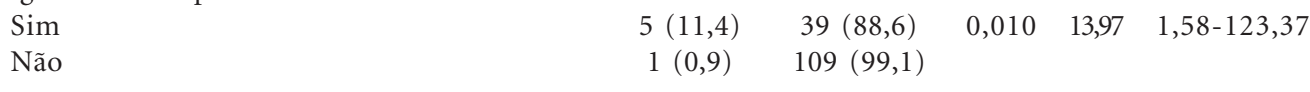

continua

gia utilizada no PEP ( $\mathrm{p}=0,009 ; \mathrm{RC}=2,64 ; \mathrm{IC}_{95 \%}$ $=1,32-5,28)$, em contraposição à dificuldade de acesso a material de referencial teórico $(\mathrm{p}=0,036$; $\left.\mathrm{RC}=0,47 ; \mathrm{IC}_{95 \%}=0,24-0,91\right)$ e à dificuldade de disponibilidade e qualidade de equipamentos e instrumentos $\left(\mathrm{p}=0,022 ; \mathrm{RC}=0,44 ; \mathrm{IC}_{95 \%}=0,23\right.$ $0,85)$, conforme mostrado na Tabela 4.

As múltiplas jornadas de trabalho, percebidas pelos supervisores como dificuldades dos médicos na Atenção Primária, também apresentaram uma associação positiva com o problema de frequência no PEP, uma vez que o fato de as múltiplas jornadas de trabalho ser considerado uma dificuldade dos médicos na APS aumenta a chance de dificuldade com frequência no PEP em 3,56 vezes $\left(\mathrm{p}=0,000 ; \mathrm{IC}_{95 \%}=1,81-7,01\right)$.

Em relação ao envolvimento dos médicos como uma dificuldade vivenciada pelos supervisores no $\mathrm{PEP}$, a escolha de temas relativos às demandas individuais ( $\mathrm{p}=0,015 ; \mathrm{RC}=0,43 ; \mathrm{IC}_{95 \%}$ $=0,22-0,82)$; aos aspectos epidemiológicos dos problemas de saúde prevalentes na APS ( $\mathrm{p}=$ 0,$\left.010 ; \mathrm{RC}=0,41 ; \mathrm{IC}_{95 \%}=0,21-0,78\right)$ e ao aprimoramento da consulta médica e dos métodos clínicos centrados no paciente $(\mathrm{p}=0,050$; $\mathrm{RC}=$ 0,$\left.42 ; \mathrm{IC}_{95 \%}=0,19-0,93\right)$, mostrou-se inversamente 
Tabela 3. continuação

\begin{tabular}{|c|c|c|c|c|c|}
\hline \multicolumn{6}{|c|}{ Assiduidade REGULAR dos médicos nas atividades do PEP } \\
\hline & $\begin{array}{l}\text { Sim } \\
\mathbf{n}(\%)\end{array}$ & $\begin{array}{l}\text { Não } \\
\text { n }(\%)\end{array}$ & $\mathbf{p}$ & $\mathrm{RC}$ & IC (95\%) \\
\hline \multicolumn{6}{|c|}{$\begin{array}{l}\text { Abordagem multidisciplinar dos problemas de } \\
\text { saúde prevalentes na APS }\end{array}$} \\
\hline Sim & $21(29,6)$ & $50(70,4)$ & 0,020 & 0,43 & $0,22-0,84$ \\
\hline Não & $41(49,4)$ & $42(50,6)$ & & & \\
\hline \multicolumn{6}{|c|}{$\begin{array}{l}\text { Atuação dos supervisores e suas dificuldades no } \\
\text { cotidiano de trabalho no PEP } \\
\text { Tempo de atuacão no PEP }\end{array}$} \\
\hline$\leq 1$ ano & $35(50,0)$ & $35(50,0)$ & 0,037 & 2,11 & $1,10-4,06$ \\
\hline$>1$ ano & $27(32,1)$ & $57(67,9)$ & & & \\
\hline \multicolumn{6}{|c|}{ Assiduidade RUIM dos médicos nas atividades do PEP } \\
\hline & $\underset{\mathbf{n}(\%)}{\operatorname{Sim}}$ & $\begin{array}{l}\text { Não } \\
\text { n }(\%)\end{array}$ & p & RC & IC $(95 \%)$ \\
\hline \multicolumn{6}{|c|}{$\begin{array}{l}\text { Tópicos abordados e atividades desenvolvidas no PEP } \\
\text { Discussão de casos clínicos }\end{array}$} \\
\hline Sim & $18(12,2)$ & $130(87,8)$ & 0,041 & 0,14 & $0,03-0,74$ \\
\hline Não & $3(50,0)$ & $3(50,0)$ & & & \\
\hline \multicolumn{6}{|c|}{$\begin{array}{l}\text { Percepção dos supervisores quanto às dificuldades } \\
\text { dos médicos na APS } \\
\quad \text { Múltiplas jornadas de trabalho }\end{array}$} \\
\hline Sim & $18(19,6)$ & $74(80,4)$ & 0,018 & 4,78 & $1,34-17,02$ \\
\hline Não & $3(4,8)$ & $59(95,2)$ & & & \\
\hline
\end{tabular}

relacionada, ou seja, a abordagem desses temas está relacionada a uma menor taxa de dificuldade com o envolvimento dos médicos (Tabela 4).

No cruzamento com as outras dificuldades percebidas pelos supervisores no PEP, o problema com o envolvimento dos médicos apresentou uma relação significativa positiva com a dificuldade de compreensão e adesão dos médicos à metodologia utilizada no PEP ( $\mathrm{p}=0,000$; $\mathrm{RC}=$ 6,$\left.13 ; \mathrm{IC}_{95 \%}=2,97-12,66\right)$ e, assim como no caso da frequência, uma relação inversa com a dificuldade de disponibilidade e qualidade de equipamentos e instrumentos ( $\mathrm{p}=0,014 ; \mathrm{RC}=0,42$; $\left.\mathrm{IC}_{95 \%}=0,22-0,81\right)$. Quanto às dificuldades na APS, observou-se novamente uma relação positiva com as múltiplas jornadas de trabalho $(\mathrm{p}=$ 0,$\left.005 ; \mathrm{RC}=2,70 ; \mathrm{IC}_{95 \%}=1,39-5,26\right)$, como observado na Tabela 4.

\section{Discussão}

Investigar a adesão dos médicos ao PEP é fundamental devido ao seu objeto de intervenção (trabalho em saúde), à amplitude geográfica de atuação, ao número de instituições e sujeitos envolvidos, ao seu custo operacional e à possibilidade de extensão para outros integrantes da ESF. A análise da adesão, a partir do olhar dos supervisores, mostrou-se de extrema relevância, dado o papel estratégico desse ator no processo de condução e mediação das discussões e atividades desenvolvidas no programa.

O trabalho em saúde, além de pressupor uma produção imaterial, consumida no momento da sua realização a partir de uma relação intersubjetiva e com uma considerável autonomia ${ }^{15}$, constitui-se em uma ação coletiva, que envolve vários sujeitos e exige domínio e interação de saberes técnico-científicos diversos. $\mathrm{Na}$ articulação entre trabalho e educação no âmbito da saúde, fazemse necessárias, tanto a compreensão de todo o processo de trabalho nesse campo, quanto a 
Tabela 4. Fatores relacionados às dificuldades vivenciadas pelos supervisores, FREQUÊNCIA e ENVOLVIMENTO dos médicos, em seu cotidiano de trabalho no PEP, Sudeste, Brasil, 2011-2012.

FREQUÊNCIA dos médicos é dificuldade do supervisor em seu cotidiano de trabalho no PEP

\begin{tabular}{|c|c|c|c|c|c|}
\hline & $\begin{array}{l}\text { Sim } \\
\mathbf{n}(\%)\end{array}$ & $\begin{array}{l}\text { Não } \\
\text { n }(\%)\end{array}$ & p & RC & IC $(95 \%)$ \\
\hline \multicolumn{6}{|c|}{$\begin{array}{l}\text { Habilidade em comunicação e educação em saúde } \\
\text { - abordagem coletiva }\end{array}$} \\
\hline Sim & $16(43,2)$ & $21(56,8)$ & 0,050 & 0,44 & $0,21-0,94$ \\
\hline Não & $74(63,2)$ & $43(36,8)$ & & & \\
\hline \multirow{2}{*}{\multicolumn{6}{|c|}{$\begin{array}{l}\text { Atuação dos supervisores e suas dificuldades no } \\
\text { cotidiano de trabalho no PEP } \\
\text { Dificuldade de acesso a material de referencial } \\
\text { teórico }\end{array}$}} \\
\hline & & & & & \\
\hline Sim & $30(47,6)$ & $33(52,4)$ & 0,036 & 0,47 & $0,24-0,91$ \\
\hline Não & $60(65,9)$ & $31(34,1)$ & & & \\
\hline \multicolumn{6}{|c|}{$\begin{array}{l}\text { Dificuldade de disponibilidade e qualidade de } \\
\text { equipamentos e instrumentos }\end{array}$} \\
\hline Sim & $30(46,9)$ & $34(53,1)$ & 0,022 & 0,44 & $0,23-0,85$ \\
\hline Não & $60(66,7)$ & $30(33,3)$ & & & \\
\hline \multicolumn{6}{|c|}{$\begin{array}{l}\text { Dificuldade de compreensão e adesão dos médicos } \\
\text { participantes à metodologia utilizada no PEP }\end{array}$} \\
\hline $\operatorname{Sim}$ & $44(72,1)$ & $17(27,9)$ & 0,009 & 2,64 & $1,32-5,28$ \\
\hline Não & $46(49,5)$ & $47(50,5)$ & & & \\
\hline \multicolumn{6}{|c|}{$\begin{array}{l}\text { Percepção dos supervisores quanto às dificuldades } \\
\text { dos médicos na APS }\end{array}$} \\
\hline \multicolumn{6}{|c|}{ Múltiplas jornadas de trabalho } \\
\hline Sim & $65(70,7)$ & $27(29,3)$ & 0,000 & 3,56 & $1,81-7,01$ \\
\hline Não & $25(40,3)$ & $37(59,7)$ & & & \\
\hline
\end{tabular}

competência para atuar com problemas de saúde e contextos socioculturais variados ${ }^{12,16-19}$. Dessa forma, o PEP pode representar um espaço de ressignificação, no qual todos os atores envolvidos assumem importância, visando uma construção coletiva de novos significados do fazer em saúde. O supervisor, entretanto, tem uma posição diferenciada a partir da função exercida de mediador e facilitador.

Ao se analisar o perfil desses sujeitos, observa-se que a diferença percentual, entre os respondentes, quanto ao gênero é pequena, o que pode indicar a tendência de mudança no perfil do médico, uma vez que, historicamente, a profissão médica apresentava um predomínio do sexo masculino. Destaca-se que no ano de 2009, o Conselho Federal de Medicina (CFM) teve mais registros do sexo feminino. No universo deste estudo, a inversão do perfil ainda não ocorreu devido, provavelmente, à maior concentração etária dos supervisores entre 31 a 40 anos, já que a maior concentração do sexo feminino, segundo o CFM, encontra-se abaixo dos 29 anos.

Além disso, o CFM demonstrou que a proporção de especialistas na área de Atenção Primária, Saúde da Família, Medicina de Família ou Saúde Pública no Brasil tem média de idade de 39,51 anos, assemelhando-se à faixa etária dos supervisores deste estudo ${ }^{20}$. Acrescenta-se que a opção por essa área justifica-se pelo campo de atuação do supervisor.

A relação supervisor/instituição de origem pode ser explicada pela distribuição das instituições para a realização do PEP, assim como por uma possível maior taxa de resposta dos supervisores vinculados à prefeitura ou pelo fato de alguns destes, mesmo ligados ao PEP das universidades, serem vinculados à rede municipal. A pequena proporção de médicos vinculados às duas instituições pode ser justificada - segundo o relatório de demografia médica no Brasil ${ }^{20}$ pelo acúmulo de vínculos em serviço público ou 
Tabela 4. continuação

ENVOLVIMENTO dos médicos é dificuldade do supervisor em seu cotidiano de trabalho no PEP

\begin{tabular}{|c|c|c|c|c|c|}
\hline & $\begin{array}{l}\text { Sim } \\
\mathbf{n}(\%)\end{array}$ & $\begin{array}{l}\text { Não } \\
\text { n }(\%)\end{array}$ & $\mathbf{p}$ & $\mathrm{RC}$ & IC (95\%) \\
\hline \multicolumn{6}{|c|}{$\begin{array}{l}\text { Critérios considerados na organização e } \\
\text { desenvolvimento dos GAP }\end{array}$} \\
\hline \multicolumn{6}{|c|}{ Demandas individuais dos médicos } \\
\hline Sim & $28(38,9)$ & $44(61,1)$ & 0,015 & 0,43 & $0,22-0,82$ \\
\hline Não & $49(59,8)$ & $33(40,2)$ & & & \\
\hline \multicolumn{6}{|c|}{$\begin{array}{l}\text { Tópicos abordados e atividades desenvolvidas no PEP } \\
\text { Aspectos epidemiológicos dos problemas de saúde } \\
\text { prevalentes na APS }\end{array}$} \\
\hline Sim & $32(39,5)$ & $49(60,5)$ & 0,010 & 0,41 & $0,21-0,78$ \\
\hline Não & $45(61,6)$ & $28(38,4)$ & & & \\
\hline \multicolumn{6}{|c|}{$\begin{array}{l}\text { Aprimoramento da consulta médica e dos } \\
\text { métodos clínicos centrados no paciente }\end{array}$} \\
\hline Sim & $55(45,5)$ & $66(54,5)$ & 0,050 & 0,42 & $0,19-0,93$ \\
\hline Não & $22(66,7)$ & $11(33,3)$ & & & \\
\hline \multicolumn{6}{|c|}{$\begin{array}{l}\text { Atuação dos supervisores e suas dificuldades no } \\
\text { cotidiano de trabalho no PEP } \\
\text { Dificuldade de disponibilidade e qualidade de } \\
\text { equipamentos e instrumentos }\end{array}$} \\
\hline Sim & $24(37,5)$ & $40(62,5)$ & 0,014 & 0,42 & $0,22-0,81$ \\
\hline Não & $53(58,9)$ & $37(41,1)$ & & & \\
\hline \multicolumn{6}{|c|}{$\begin{array}{l}\text { Dificuldade de compreensão e adesão dos médicos } \\
\text { participantes à metodologia utilizada no PEP }\end{array}$} \\
\hline Sim & $46(75,4)$ & $15(24,6)$ & 0,000 & 6,13 & $2,97-12,66$ \\
\hline Não & $31(33,3)$ & $62(66,7)$ & & & \\
\hline \multirow{2}{*}{\multicolumn{6}{|c|}{$\begin{array}{l}\text { Percepção dos supervisores quanto às dificuldades } \\
\text { dos médicos na APS }\end{array}$}} \\
\hline Múltiplas jornadas de trabalho & & & & & \\
\hline Sim & $55(59,8)$ & $37(40,2)$ & 0,005 & 2,70 & $1,39-5,26$ \\
\hline Não & $22(35,5)$ & $40(64,5)$ & & & \\
\hline
\end{tabular}

privado, com a atividade em consultório pela maioria dos médicos. Ainda, o grande percentual dos supervisores com um maior tempo no PEP demonstra que eles têm experiências mais significativas na APS, parecendo traduzir um horizonte mais ampliado e rico, possibilitando melhor condução do processo de supervisão e maior estímulo para uma participação mais efetiva dos médicos no programa.

A precariedade do espaço físico destinado às atividades do PEP pode representar o pequeno interesse da gestão em tornar a estratégia educativa mais acolhedora, o que induz a questionamentos quanto à qualidade da ação educacional, ou seja, a adequação dos recursos institucionais à proposta é fundamental para o pleno desenvolvimento da ação educativa, assim como evidenciado no projeto para conformação da Rede de Polos de Educação Permanente em Saúde da
Bahia $^{21}$. A adequação do espaço físico às demandas dos GAP não considerada ruim está relacionada à boa participação dos médicos, assim como a boa adequação do espaço está associada à ótima participação e à assiduidade dos médicos. Esse resultado evidencia uma estreita relação entre o espaço físico e a adesão dos médicos ao PEP. Uma boa adequação do espaço, portanto, parece influenciar a adesão dos médicos, ou uma boa participação dos médicos promove maiores investimentos no espaço utilizado nos GAP. Definir qual das duas está associada ao PEP exige o desenvolvimento de estudos qualitativos.

Os tópicos abordados no PEP foram as variáveis que mais apresentaram associação com a adesão dos médicos ao programa. A abordagem multidisciplinar dos problemas de saúde prevalentes na APS se associou positivamente à ótima participação dos médicos e a abordagem dos as- 
pectos epidemiológicos prevalentes na APS se relacionou com a ótima participação e ótima assiduidade dos médicos. Essa situação demonstra que o PEP tem buscado abordar o contexto da APS, minimizando a concepção da qualificação dos profissionais das ESF como elemento crítico.

A abordagem interdisciplinar do trabalho em equipe multiprofissional tem sido evidenciada como um processo voltado ao cuidado integral das necessidades de saúde da população, além do âmbito individual-biológico, e à melhora da qualidade de vida da comunidade. Médicos residentes de uma residência multiprofissional relatam que os momentos de discussão e trocas de experiências com os outros profissionais potencializam a construção do conhecimento e a articulação com as outras áreas do saber ${ }^{22}$.

Por outro lado, a não abordagem multidisciplinar e a não discussão de eventos críticos se mostraram relacionadas à participação regular dos médicos, e a falta de enfoque epidemiológico dos problemas prevalentes na APS se associou tanto à participação regular dos médicos quanto à dificuldade de envolvimento dos mesmos nas atividades. Essa realidade parece estar associada à estrutura da graduação em Medicina, a qual contempla de maneira frágil as questões das ciências sociais, do planejamento e da epidemiologia, tripé disciplinar da Saúde Coletiva ${ }^{23}$.

Além disso, no âmbito da Educação Médica, as abordagens multidisciplinares; epidemiológicas; do impacto das políticas de saúde nos cuidados voltados à comunidade e ao paciente; da economia em saúde e da não centralização na doença e na média e alta complexidade não são realizadas de maneira adequada. Enfatiza-se ainda a importância de enfoques que ressaltam a interação com a comunidade e a gestão local do SUS, configurando uma formação menos hierarquizada e que aproxima os diversos sujeitos na prática em saúde. Assim, provavelmente, os profissionais com um perfil diferenciado, inseridos em unidades de APS melhor estruturadas, tentam, de alguma maneira compensar esta fragilidade nesse processo de qualificação, o que pode favorecer a fixação deste médico na $\mathrm{ESF}^{18,24,25}$.

A abordagem da habilidade em comunicação e educação em saúde com abordagem individual/relação médico-usuário apresentou uma relação positiva com a participação considerada regular dos médicos no programa. A discussão de tópicos envolvendo habilidade em comunicação e educação em saúde focada na abordagem coletiva apresentou uma relação inversa com a dificuldade de frequência dos médicos evidenci- ando que a abordagem da educação em saúde centrada na abordagem coletiva está relacionada à melhor adesão dos médicos. $\mathrm{O}$ processo de trabalho é regido pela ação comunicativa e a prática em saúde depende da relação estabelecida entre a equipe de saúde e os usuários, favorecendo a interação entre promoção, prevenção e recuperação da saúde e, dessa forma, conseguindo compreender as necessidades de saúde da comunidade adscrita. O Programa, portanto, é um processo educativo de características emancipatórias, uma vez que consegue envolver os médicos para ações coletivas e participativas, facilitando o foco no princípio da integralidade ${ }^{26}$.

A assiduidade ruim dos médicos apresentou uma relação inversa com a discussão de casos clínicos no PEP. A escolha de temas relativos às demandas individuais dos profissionais para o aprimoramento da consulta médica e dos métodos clínicos centrados na perspectiva dos residentes médicos em saúde da família apresentaram uma associação inversa com a dificuldade de envolvimento dos médicos. Essa posição demarca que os médicos participantes buscam ultrapassar o modelo de educação tradicional, próximo ao da graduação que privilegia a concepção biomédica centrada na abordagem individual, na consulta, na cura da doença e no trabalho isolado. A discussão de casos clínicos é reconhecida como espaço de experiência do cotidiano laboral dos profissionais da ESF, abrindo possibilidades para a realização de ações em saúde com a participação efetiva dos profissionais no planejamento ${ }^{26,27}$.

Em relação às dificuldades vivenciadas pelos supervisores, foi encontrada uma associação estatisticamente significativa entre a ótima assiduidade dos médicos e o fato de a compreensão e adesão dos médicos participantes à metodologia utilizada no PEP não ser uma dificuldade vivenciada pelo supervisor. A estratégia metodológica utilizada nas ações educativas é pautada em metodologias ativas de ensino-aprendizagem, que privilegiam a participação ativa dos sujeitos, a articulação entre teoria e prática e a problematização da realidade. Os trabalhadores participam efetivamente na construção de novas abordagens do 'fazer' em saúde e dos temas a serem contemplados nos CAPP, de acordo com as necessidades de qualificação, além de ter o supervisor como facilitador e orientador desse processo. A assimilação e a incorporação da metodologia do programa pelos médicos são fundamentais para a consolidação do progra$\mathrm{ma}$, assim como evidenciado na literatura ${ }^{27}$.

Simultaneamente, essa dificuldade mostrou uma relação positiva com a participação consi- 
derada regular dos médicos no programa e com as dificuldades de frequência e de envolvimento dos médicos, apontando ser um fator relevante para a adesão ao PEP.

No caso da participação ruim dos médicos nas atividades do PEP, observou-se uma relação estatisticamente positiva com a dificuldade de articulação e cooperação por parte da gestão municipal. Apesar do reconhecimento em relação à importância do investimento em educação permanente para os trabalhadores da Saúde da Família, tanto para o desenvolvimento de competências e de habilidades complexas e diversificadas, quanto para a mudança nas práticas, a literatura relata baixa participação desses trabalhadores nas ações educativas, muitas vezes devido à falta de pessoal, o que impede muitos profissionais de saírem de suas unidades para realizar essas capacitações ${ }^{28}$. Neste estudo, a falta de cooperação do gestor municipal pode estar reproduzindo uma preocupação com a permanência do profissional no local, com a redução da produtividade e com o não reconhecimento do programa como um excelente mecanismo de organização do trabalho na APS.

A dificuldade de acesso ao referencial teórico, por outro lado, mostrou-se inversamente relacionada à dificuldade de frequência dos médicos, demonstrando a participação nos CAPP como uma forma de minimizar os efeitos do problema de acesso ao material. A dificuldade quanto à disponibilidade e qualidade de equipamentos e instrumentos, no entanto, relacionou-se a uma menor dificuldade no envolvimento dos profissionais. Destaca-se que as condições de trabalho devem ser adequadas para o desempenho das funções, desde uma boa organização, estrutura física e de material e equipamentos apropriados, seguros e disponíveis para que estimulem a fixação dos profissionais e o alcance de resultados efeti$\operatorname{vos}^{18,29}$.

Quanto às dificuldades dos médicos na APS percebidas pelos supervisores, observou-se uma associação significativa entre a ótima assiduidade e a remuneração insatisfatória dos médicos. Esse resultado pode ser explicado pela necessidade dos médicos de interação com seus pares para discussão dos problemas vivenciados pela categoria, como a remuneração insatisfatória. A utilização desses espaços é uma maneira de fortalecer e legitimar diversas ações por parte dos profissionais, desde condições inadequadas de trabalho, até a melhoria nos salários.

Algumas estratégias, conforme relatado na literatura, têm sido adotadas pelas Secretarias
Municipais de Saúde (SMS) diante das reivindicações e movimentos dos trabalhadores das ESF visando remunerações mais condizentes com as suas funções e responsabilidades ${ }^{18}$. As autoras estudaram o processo de gestão do trabalho no âmbito das equipes em quatro municípios brasileiros, sendo que dois desses criaram formas de melhor remunerar os integrantes das equipes. Uma dessas melhorias trouxe questionamentos, uma vez que a carga horária dos trabalhadores em regime estatutário foi fixada em vinte horas, somadas a mais vinte horas na forma de extensão de jornada de trabalho, gerando prejuízos aos direitos trabalhistas, inclusive na aposentadoria. Outra medida relatada foi a disponibilização pela SMS de plantões em pronto-atendimento/urgência para os profissionais das equipes, com uma remuneração adicional ${ }^{18}$.

As múltiplas jornadas de trabalho, percebidas pelos supervisores como dificuldades dos médicos na Atenção Primária, apresentaram uma associação positiva, tanto com a assiduidade ruim dos médicos, quanto com o problema de frequência e de envolvimento dos médicos no PEP. Tal achado aponta que o problema das múltiplas jornadas na Medicina - uma realidade evidenciada na literatura $\mathrm{a}^{30,31}$ - pode estar reduzindo o tempo disponível dos médicos para participarem do Programa.

Apesar de a profissão médica lutar pela manutenção de uma prática liberal, os profissionais se submetem às regras do sistema capitalista moderno, caracterizado por múltiplos vínculos trabalhistas; pelo ritmo acelerado, sobrecarregado e prolongado nas jornadas de trabalho; pela instabilidade e pela baixa autonomia, em busca de uma remuneração ideal. O multiemprego na categoria médica geralmente é marcado pela simultaneidade de práticas assalariadas, tanto públicas, como privadas, com a liberal. Esse contexto favorece o não envolvimento adequado às questões relativas às instituições as quais estão vinculados e uma frágil inserção no âmbito do processo de atenção à saúde, o que favorece a precarização do SUS ${ }^{30,31}$.

A boa adesão ao PEP parece se relacionar à possibilidade que o programa apresenta de se construir coletivamente, a partir de significados e valores apreendidos na realidade laboral cotidiana e abordagem do médico no processo de atenção à saúde ${ }^{32}$. Para isso, pressupõe-se uma ação/reflexão crítica e problematizada, além da colaboração para a minimização dos desafios apresentados relacionados à baixa adesão. A centralização da qualificação em uma determinada 
categoria, entretanto, impede uma compreensão mais abrangente da realidade cotidiana do processo de trabalho na ESF, podendo se perder pelo caminho uma série de significados e valores ${ }^{33,34}$.

Apesar dos desafios evidenciados, o PEP busca resgatar habilidades e competências, conhecimentos e saberes, a partir do conhecimento técnico e científico, das experiências de trabalho e de interação social no 'fazer' médico. O programa compreende que alguns dos critérios para se ter uma APS de qualidade e caracterizada como o centro de comunicação entre os pontos de atenção, em uma perspectiva de horizontalidade, é a resolutividade e a credibilidade dos médicos quanto ao diagnóstico e à terapêutica, a redução de encaminhamentos à média e alta complexidade, mas, ao mesmo tempo, uma melhor interação entre os pontos de atenção, o usuário e a comunidade.

O desafio de qualificar os profissionais para o processo de trabalho no SUS vem sendo cumprido, atualmente, em cem por cento do Estado investigado, com o apoio da própria gestão estadual, das instituições parceiras e dos sujeitos envolvidos no processo. Acrescenta-se, ainda, o estímulo que o programa traz para ampliar a sua discussão, enquanto estratégia, a partir da abertura para a realização de uma investigação que visa, primordialmente, apreender suas implicações e as necessidades de reconstrução do programa.

\section{Considerações finais}

Espera-se que este estudo possa contribuir para o desenvolvimento do programa e para as discussões sobre as políticas e ações em Educação Permanente direcionadas ao profissional de saúde. As vantagens do estudo se encontram na possibilidade de generalização dos resultados para o Estado, dada a alta taxa de resposta apresentada e o tipo de informações obtidas (objetivas e voltadas para o delineamento de um panorama geral do trabalho realizado pelos supervisores no PEP). As desvantagens, por sua vez, estão relacio- nadas à não abordagem de características específicas de cada instituição e/ou situação, à não inclusão de outros atores envolvidos, além da impossibilidade de se aprofundar questões relativas aos microprocessos de trabalho no programa, o que seria acessado por meio de um estudo qualitativo.

\section{Colaboradores}

LS D’Ávila contribuiu substancialmente para a coleta, concepção, planejamento e análise das informações; participou da revisão bibliográfica para discussão dos resultados; contribuiu significativamente na elaboração do artigo e na revisão crítica do conteúdo; e participou da aprovação da versão final do manuscrito. LN Assis contribuiu substancialmente para a coleta, concepção, planejamento e análise das informações; contribuiu significativamente na elaboração do artigo e na revisão crítica do conteúdo; e participou da aprovação da versão final do manuscrito. MB Melo participou da revisão bibliográfica para discussão dos resultados; contribuiu significativamente na elaboração do artigo e na revisão crítica do conteúdo; e participou da aprovação da versão final do manuscrito. LC Brant contribuiu significativamente na elaboração do artigo e na revisão crítica do conteúdo; e participou da aprovação da versão final do manuscrito. 


\section{Referências}

1. Brasil. Ministério da Saúde (MS). Programa de Saúde da Família: ampliando a cobertura para consolidar a mudança do modelo de atenção básica. Brasília: MS; 2003.

2. Leão CDA, Caldeira AP. Avaliação da associação entre qualificação de médicos e enfermeiros em atenção primária em saúde e qualidade da atenção. Cien Saude Colet 2011; 16(11):4415-4423.

3. Brasil. Ministério da Saúde (MS). Portaria GM/MS $\mathrm{n}^{\circ}$ 1.996, de 20 de agosto de 2007. Dispõe sobre as diretrizes para a implementação da Política Nacional de Educação Permanente em Saúde e dá outra providências. Diário Oficial da União 2007; 22 ago

4. Silvério JB. Programa de educação permanente para médicos de família. Rev Med Minas Gerais 2008; 18(Supl. 4):S60-S66.

5. Cavalcanti YW, Wanzeler MCC. Educação Permanente em Saúde na Qualificação de Processos de Trabalho em Saúde Coletiva. Rev Bras Cien Saude 2009; 13(1):13-20.

6. Ceccim RB, Feuerwerker LCM. O quadrilátero da formação para a área da saúde: ensino, gestão, atenção e controle social. Physis: Rev Saude Colet 2004; 14(1):41-65.

7. Brasil. Ministério da Saúde (MS). Secretaria de Gestão do Trabalho e da Educação na Saúde. Departamento de Gestão da Educação em Saúde. Política Nacional de Educação Permanente em Saúde. $1^{\text {a }}$ Edição. Brasília: MS; 2009.

8. Merhy EE. A rede básica como uma construção da saúde pública e seus dilemas. In: Merhy EE, Onocko R, organizadores. Agir em saúde: um desafio para o público. São Paulo, Buenos Aires: Hucitec, Lugar Editorial; 1997.

9. Silva LAA, Ferraz F, Lino MM, Backes VMS, Schmidt SMS. Educação permanente em saúde e no trabalho de enfermagem: perspectiva de uma práxis transformadora. Rev Gaucha Enferm 2010; 31(3): 557-561.

10. Ezequiel MCDG, Noel BK, Lemos PP, Paiva AC, Borges LP, Ferreira GM, Sanzovo PS. Estudantes e usuários avaliam ferramenta de educação permanente em saúde - Sieps. Rev Bras Educ Med 2012; 36(1; Supl. 2):112-130.

11. Heimann LS, Ibanhes LC, Boaretto RC, Castro IEN, Telesi Júnior E, Cortizo CT, Fausto MCR, Nascimento VB, Kayano J. Atenção primária em saúde: um estudo multidimensional sobre os desafios e potencialidades na Região Metropolitana de São Paulo (SP, Brasil). Cien Saude Colet 2011; 16(6): 2877-2887.

12. Starfield B. Atenção primária: equilíbrio entre necessidades de saúde, serviços e tecnologia. Brasília: Unesco, Ministério da Saúde (MS); 2002.

13. Wyatt JC. When to use web-based surveys. Editorial Comment. J Am Med Inform Assoc 2000; 7(4):426429.

14. Archer TM. Response rates to expect from webbased surveys and what to do about it. J Exten [serial on Internet] 2008 June [cited 2012 Jan 25]; 46(3): Available from: http://www.joe.org/joe/2008june/ rb3.php
15. Martins MIC, Dal Poz MR. A qualificação de trabalhadores de saúde e as mudanças tecnológicas. Physis: Rev Saude Colet 1998; 8(2):125-146.

16. Peduzzi M, Del Guerra DA, Braga CP, Lucena FS, Silva JAM. Atividades educativas de trabalhadores na atenção primária: concepções de educação permanente e de educação continuada em saúde presentes no cotidiano de Unidades Básicas de Saúde em São Paulo. Interface (Botucatu) 2009; 13(30):121134.

17. Pinheiro R, Mattos RA, organizadores. Gestão em redes: práticas de avaliação, formação e participação na saúde. Rio de Janeiro: CEPESC; 2006.

18. Mendonca MHM, Martins MIC, Giovanella L, Escorel S. Desafios para gestão do trabalho a partir de experiências exitosas de expansão da Estratégia de Saúde da Família. Cien Saude Colet 2010; 15(5):23552365.

19. Tesser CD, Garcia AV, Vendruscolo C, Argenta CE. Estratégia saúde da família e análise da realidade social: subsídios para políticas de promoção da saúde e educação permanente. Cien Saude Colet 2011; 16(11):4295-4306.

20. Brasil. Conselho Federal de Medicina. Demografia Médica no Brasil. São Paulo, Brasília: Conselho Regional de Medicina do Estado de São Paulo, Conselho Federal de Medicina; 2011.

21. Silva Pinto LL, Barboza Filho JC. Considerações sobre o projeto para conformação da Rede de Polos de Educação Permanente em Saúde da Bahia. Rev Baiana Saude Publica 2005; 29(1):126-135.

22. Ferreira RC, Varga CRR, Silva, RF. Trabalho em equipe multiprofissional: a perspectiva dos residentes médicos em saúde da família Cien Saude Colet 2009; 1(Supl. 1):1421-1428.

23. Paim JS, Almeida Filho N. A crise da Saúde Pública e a utopia da Saúde Coletiva. Salvador: Casa da Qualidade Editora; 2000.

24. Kesselheim AS, Kirsten E, Austad BS. Residents: Workers or Students in the Eyes of the Law? N Engl $J$ Med 2011; 364(8):695-697.

25. Oliveira NA, Alves LA. Ensino médico, SUS e início da profissão: como se sente quem está se formando? Rev Bras Educ Med 2011; 35(1):26-36.

26. Silva JAM, Peduzzi M. Educação no Trabalho na Atenção Primária à Saúde: interfaces entre a educação permanente em saúde e o agir comunicativo. Saude Soc 2011; 20(4):1018-1032.

27. Cotrim-Guimarães IMA. Programa de educação permanente e continuada da equipe de enfermagem da clínica médica do Hospital Universitário Clemente de Faria: análise e proposições. [dissertação]. Rio de Janeiro (RJ): Escola Nacional de Saúde Pública Sergio Arouca; 2009.

28. Shimizu HE, Reis LS. As representações sociais dos trabalhadores sobre o Programa Saúde da Família Cien Saude Colet 2011; 16(8):3461-3468.

29. Goncalves RJ, Soares RA, Troll T, Cyrino EG. Ser médico no PSF: formação acadêmica, perspectivas e trabalho cotidiano. Rev Bras Educ Med 2009; 33(3):382-392. 
30. Machado MH. Programa de Saúde da Família no Brasil algumas considerações sobre o perfil dos médicos e enfermeiros. In: Sousa MF, organizador. Os sinais vermelhos do PSF. São Paulo: Hucitec; 2002.

31. Maciel RH, Santos JBF, Sales TB, Alves MAA, Luna, AP, Feitosa LB. Multiplicidade de vínculos de médicos no Estado do Ceará. Rev Saude Publica 2010; 44(5):950-956.

32. Ayres JRC. Cuidado e reconstrução das práticas de saúde. Interface (Botucatu) 2004; 8(14):73-91.

33. Consórcio Medicina USP. Recursos humanos na atenção básica, estratégias de qualificação e Pólos de Educação Permanente no Estado de São Paulo. São Paulo: Centro de Estudos de Cultura Contemporânea; 2008.

34. Batista KBC, Gonçalves OSJ. Formação dos profissionais de saúde para o SUS: significado e cuidado. Saude Soc 2011; 20(4):884-899.

Artigo apresentado em 18/03/2013

Aprovado em 13/06/2013

Versão final apresentada em 27/06/2013 